\title{
WICO Graph: a Labeled Dataset of Twitter Subgraphs based on Conspiracy Theory and 5G-Corona Misinformation Tweets
}

\author{
Daniel Thilo Schroeder ${ }^{12} \mathbb{( D}^{\mathrm{a}}$, Ferdinand Schaal ${ }^{3} \mathbb{D}^{\mathrm{b}}$, Petra Filkukova ${ }^{4} \mathbb{C l}^{\mathrm{c}}$, \\ Konstantin Pogorelov ${ }^{4}{ }^{\mathrm{d}}$ and Johannes Langguth ${ }^{4}{ }^{\mathrm{e}}{ }^{\mathrm{e}}$ \\ ${ }^{1}$ Simula Metropolitan Center for Digital Engineering, Oslo, Norway \\ ${ }^{2}$ Technical University of Berlin, Germany \\ ${ }^{3}$ Technical University of Denmark, Denmark \\ ${ }^{4}$ Simula Research Laboratory, Fornebu, Norway \\ s145434@student.dtu.dk, \{daniels, petrafilkukova, konstantin, langguth $\} @$ simula.no
}

Keywords: Graph Neural Networks, Graph Algorithms, Misinformation, Fake-News Detection.

\begin{abstract}
In the wake of the COVID-19 pandemic, a surge of misinformation has flooded social media and other internet channels, and some of it has the potential to cause real-world harm. To counteract this misinformation, reliably identifying it is a principal problem to be solved. However, the identification of misinformation poses a formidable challenge for language processing systems since the texts containing misinformation are short, work with insinuation rather than explicitly stating a false claim, or resemble other postings that deal with the same topic ironically. Accordingly, for the development of better detection systems, it is not only essential to use hand-labeled ground truth data and extend the analysis with methods beyond Natural Language Processing to consider the characteristics of the participant's relationships and the diffusion of misinformation. This paper presents a novel dataset that deals with a specific piece of misinformation: the idea that the $5 \mathrm{G}$ wireless network is causally connected to the COVID-19 pandemic. We have extracted the subgraphs of 3,000 manually classified Tweets from Twitter's follower network and distinguished them into three categories. First, subgraphs of Tweets that propagate the specific 5G misinformation, those that spread other conspiracy theories, and Tweets that do neither. We created the WICO (Wireless Networks and Coronavirus Conspiracy) dataset to support experts in machine learning experts, graph processing, and related fields in studying the spread of misinformation. Furthermore, we provide a series of baseline experiments using both Graph Neural Networks and other established classifiers that use simple graph metrics as features. The dataset is available at https://datasets.simula.no/wico-graph.
\end{abstract}

\section{INTRODUCTION}

In large parts of the world, the COVID-19 pandemic is likely the most impactful event for decades, and it has posed unprecedented challenges for affected countries and their governments. Consequently, coverage of the pandemic has dominated international news for months. At the same time, such massive amounts of misinformation have circulated online that the term "infodemic" was coined to describe the phenomenon [Ali and Kurasawa, 2020, Ghebreyesus and

\footnotetext{
a (iD https://orcid.org/0000-0000-0000-0000

b (iD https://orcid.org/0000-0000-0000-0000

c (iD https://orcid.org/0000-0003-4256-4501

d(D) https://orcid.org/0000-0000-0000-0000

e (iD https://orcid.org/0000-0003-4200-511X
}

$\mathrm{Ng}, 2020]$. It is widely suspected that some of it is disinformation specifically targeted at western democracies [European External Action Service (EEAS), 2020]. Whether created intentionally or by mistake, the misinformation has had severe consequences. For example, in January 2020, when COVID-19 was still restricted to Wuhan, China, the first Tweet linking the outbreak to $5 \mathrm{G}$ wireless technology appeared on Twitter. While these Tweets got a little initial reaction, about ten weeks later, in early April, a series of arson attacks hit $5 \mathrm{G}$ cell towers in the UK and other countries. Thus, seemingly inconsequential misinformation had become a digital wildfire. Digital wildfires, i.e., fast-spreading and inaccurate, counterfactual, or intentionally misleading information that quickly permeates public consciousness and has severe real-world implications, have been placed among 
the top global risks in the 21 st century by the World Economic Forum [Howell, 2013]. While a sheer endless amount of misinformation exists on the internet, only a small fraction of it spreads far and affects people to a degree where they commit harmful or criminal acts in the real world. Thus, detecting such digital wildfires with the aim of providing factual information to counter the misinformation is an important goal. However, due to the vast volume of social media postings, with 500 million daily tweets on Twitter alone, manual oversight is impossible. Thus, it is necessary to devise systems that can automatically detect misinformation narratives.

Typically, developing such systems requires large amounts of labeled input data. Hence, this contribution aims to provide such data, along with human annotations concerning the contents of the tweets. A wide variety of technical approaches to the problem, which is often referred to as fake news detection, have been developed over the past years [de Beer and Matthee, 2020]. Depending on the chosen approach, different data is required. This paper presents a dataset for graph-based detection methods that recognize misinformation based on its spreading patterns or the spreader's social network.

Our dataset focuses on misinformation related to COVID-19 and 5G wireless technology. The reason for this choice was the fact that it developed into a digital wildfire. Furthermore, the topic contains predominantly statements such as " $5 \mathrm{G}$ radiation causes corona" that contradict established scientific consensus. Thus, unlike in the case of more political statements, we assume an extensive agreement w.r.t. what we labeled as misinformation.

We obtained the data using Twitter's search API by looking for Tweets containing 5G, Corona, and related terms. The dataset consists of 3,492 subgraphs of Twitter's follower network. We labeled each subgraph as belonging to three classes: promoting $5 \mathrm{G}$ conspiracy, promoting another conspiracy, and not promoting conspiracies.

The remainder of the paper describes how the dataset was collected and according to which criteria the labels were assigned. Furthermore, we introduce the dataset's general characteristics and provide baseline classification accuracy, which we obtained via a relatively straightforward application of graph neural networks, specifically graph isomorphism networks [Xu et al., 2018] as well as Random Forest [Breiman, 2001] and Naive Bayes classification using graph metrics such as the clustering coefficient as features.

\section{RELATED WORK}

The utility of automated fake news detection, as well as the necessity of labeled datasets that comes with it, has been widely recognized in the recent past. A recent survey [de Beer and Matthee, 2020] divides misinformation detection mechanisms into four categories: language based [Burkhardt, 2017, Yang et al., 2018], topic agnostic [Horne and Adali, 2017, Castelo et al., 2019], machine learning based [Pérez-Rosas et al., 2018, Sivasangari et al., 2018], and knowledge based approaches [Hassan et al., 2017, Ahmed et al., 2019]. The authors recognize that some approaches transcend these categories and thus classify them as hybrid. In addition, some approaches to the problem also aim at clustering fake news into categories or topics [Zhang et al., 2019, Hosseinimotlagh and Papalexakis, 2018].

Their categorization does not distinguish between the different machine learning (ML) methods and associate learning techniques such as deep learning. However, the wide variety of the field makes it necessary to further subdivide the category. Modern approaches to the problem include neural [Le et al., 2020] and graph attention networks [Cui et al., 2020]. In this work, we are primarily targeting graph-based approaches that learn the spreading structure of misinformation rather than trying to identify its contents. Such methods have also been applied in the closely related topic of rumor detection [Ma et al., 2019, Huang et al., 2019].

In addition to the methods, a variety of misinformation datasets have recently been presented [Wang, 2017, Salem et al., 2019, Dhoju et al., 2019, Dai et al., 2020, Shu et al., 2018, Ghenai and Mejova, 2018, Cui and Lee, 2020]. Cui and Lee [Cui and Lee, 2020] give a comprehensive overview over the characteristics of the different datasets. However, these datasets are focused on contents rather than graph structure. Datasets for testing graph comparison methods exist [Kersting et al., 2016], but they are generally not based on misinformation. Our contribution aims to close this gap. Some approaches to the problem also aim at clustering fake news into categories or topics [Zhang et al., 2019, Hosseinimotlagh and Papalexakis, 2018].

\section{5G CONSPIRACY INSIGHTS}

As mentioned in the introduction, the $5 \mathrm{G}$ corona conspiracy theory refers to a causal relationship between the 5G mobile phone standard and the COVID19 virus, reflected in several narratives. For exam- 
ple, there is the assumption that $5 \mathrm{G}$ radiation weakens the immune system or that the expansion of the $5 \mathrm{G}$ network and the virus allegedly associated with it is part of a Chinese plan to attack the West. From January 21,2020 , until the end of the month, we could observe 685 tweets and 1080 retweets distributed simultaneously with YouTube videos containing similar content. During March, the number of tweets grew rapidly and reached a peak between March 20 and April 4. The rapid increase is due to our assessment after distributing a series of videos in the UK containing 5G Corona narratives, which were distributed from March 25 onwards. In mid to late April, both Twitter and YouTube have decided not to allow any content related to any of the narratives that refer to the $5 \mathrm{G}$ corona conspiracy theory.

\section{TWITTER IN A NUTSHELL}

Twitter is a short message service that enables posting messages of up to 280 characters. Hereafter we will use the term status as a generic term for all messages on Twitter. Statuses may be either public or only available to users in a follower-friend relationship with the author. A status is called a tweet if it is not directly related to another status. Tweets are shown to the author's followers and can be commented on by any other Twitter user. Comments on Tweets are then called replies. Because every reply itself can be commented, it is possible to create nested structures called threads. Furthermore, both tweets and replies are shareable. Depending on whether a status is commented when sharing, the shared status is called either a quote or a retweet. Since the content of a retweet corresponds to the shared status, the scope of interpretation transmits in equal measure and, therefore, allows us to understand retweets as a form of agreement. For each tweet, quotes, and reply, we build subgraph which contains the status itself and its retweets. Since it is also possible to retweet a reply, we treat the reply as both part of the original subgraph and also the as first status of a new subgraph.

\section{DATA COLLECTION}

We collected about one billion statuses that included keywords related to the COVID-19 crisis from Twitter between January 17, 2020 and Mai 15, 2020 using the fact framework [Schroeder et al., 2019] for Twitter data collection [Authors blinded]. In a second step, we selected statuses that mention 5G in any conceivable spelling such as $5 \mathrm{G}, 5 \mathrm{~g}$, or $\# 5 \mathrm{~g}$, resulting
Table 1: The number of statuses ordered by the types (section 4). All numbers shown in this table refer to the statuses after thread recovery. A user can be in more than one category simultaneously.

\begin{tabular}{|c|c|c|}
\hline & initial & selected \\
\hline \multicolumn{3}{|c|}{ Overall } \\
\hline Sources & 177498 & 3492 \\
\hline Retweets & 351848 & 205097 \\
\hline Users & 413885 & 159104 \\
\hline Deleted users & 11788 & 3821 \\
\hline Collection & \multicolumn{2}{|c|}{$17 / 01 / 2020$} \\
\hline Collection & \multicolumn{2}{|c|}{$15 / 05 / 2020$} \\
\hline \multicolumn{3}{|c|}{$5 \mathrm{G}$} \\
\hline Sources & & 406 \\
\hline Retweets & & 18588 \\
\hline Users & & 14792 \\
\hline earliest & & $25 / 01 / 2020$ \\
\hline latest & & $08 / 05 / 2020$ \\
\hline \multicolumn{3}{|c|}{ Other } \\
\hline Sources & & 596 \\
\hline Retweets & & 32015 \\
\hline Users & & 38095 \\
\hline earliest & & $11 / 06 / 2012$ \\
\hline latest & & $08 / 05 / 2020$ \\
\hline \multicolumn{3}{|c|}{ Non } \\
\hline Sources & & 2490 \\
\hline Retweets & & 154494 \\
\hline Users & & 128139 \\
\hline earliest & & $28 / 03 / 2014$ \\
\hline latest & & $09 / 05 / 2020$ \\
\hline
\end{tabular}

in a set of 364,325 statuses that potentially include text related to 5G-Corona conspiracy theories. Although this approach may seem simple, it was very successful in this case since $5 \mathrm{G}$ is a specific term with little semantics not pointing to the standard for cellular networks. Furthermore, since all statuses covering 5G-Corona conspiracy theories are of interest and not only the ones containing a keyword, we restored the threads that contain the statuses we found using the text search. However, the Twitter API only allows us to query parent elements starting from a given status, so restoring is only feasible for statuses posted earlier than the ones we found using the text search. Later parts of the threads cannot be found in this manner.

After completing the threads, the number of candidates for statuses related to 5G-Corona conspiracy theories increased to 801,515 . Since sharing a retweet is the same as sharing the original status, we removed the retweets before selecting 10,000 tweets randomly for manual labeling using the classes described in Section 6. Furthermore, as most tweets that were labeled as misinformation had very few retweets, we identified statuses with associated subgraphs in order 


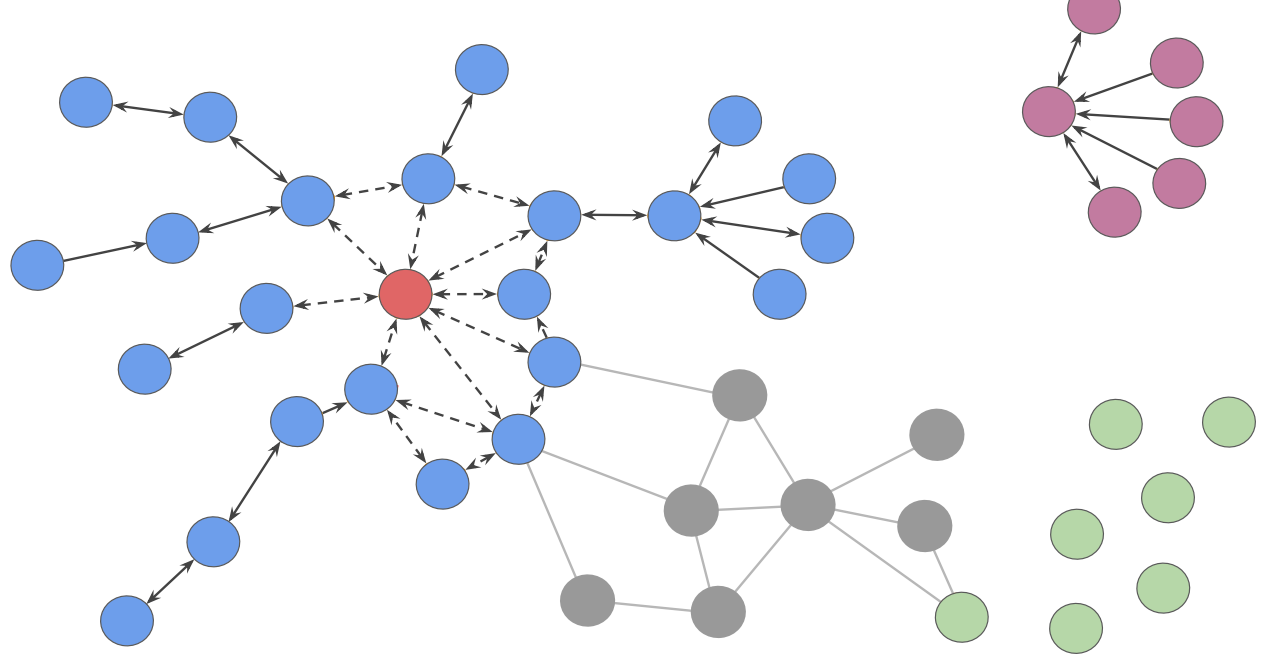

Figure 1: The Figure shows an example of the induced subgraph of a single status where nodes represent Twitter users. Follower relationships connect to users and can be bidirectional. The red node represents the source status author, while the purple nodes stage a connected component with no connection to the main component, as discussed in Section 7. This happens when content propagates outside of the Twitter follower network. Green nodes correspond to users that retweeted without a path to the source and followers in the subgraph. We assume that both purple and green nodes, as well as their relationships to either each other or the main component, can be used to distinguish the classes (see Section 6). Beyond that, the dotted edges mark unclear distribution channels, i.e. it is not clear which edge was responsible for spreading the message. However, some of these ambiguous edges could be removed with the help of timestamps. Finally, the grey nodes represent users who were not involved in distributing the status. This implies that even though there is a path from the red to the green node, information traveled outside the network.

to enhance the dataset. Table 1 lists the number of statues obtained from each class, along with supporting information such as the total number of user accounts in the subgraphs and the number of accounts that were deleted at the end of the data collection. Interestingly over $90 \%$ of the deleted users profiles were created in 2020. Note that the tweets that were posted before the collection began are a result of restoring the threads.

\section{CLASS LABELING}

From the collected data we created a dataset of manually labeled statuses. The labeling was performed by a diverse group of staff scientists, postdocs, and graduate students. The primary criterion for our classification was the potential spread of misinformation. Our definition of spreading requires that the author gives the impression of at least partially believing the presented misinformation. This includes statements that present the misinformation as uncertain, e.g., statements such as: "Does $5 G$ cause COVID-19?" assuming there is no additional information marking the claim as highly unlikely. The rationale for this is that by presenting such an idea as a valid hypothesis, a tweet essentially spreads misinformation by implying that the suggested idea could be considered possible by established science. On the other hand, we do not consider statements that merely point out that certain misinformation exists to be spreading that misinformation since our focus lies on detecting intentions contained in the wording. To this end, we classified statuses into three categories:

\section{G Conspiracy}

This class contains the subgraphs of statuses that claim or insinuate some more profound connection between COVID-19 and 5G, such as the idea that $5 \mathrm{G}$ weakens the immune system and thus caused the pandemic, or that there is no pandemic and radiation emitted by $5 \mathrm{G}$ network towers harmed the presumed COVID-19 victims. The crucial requirement is the claimed existence of some causal link. There are 406 subgraphs in this category.

\section{Other Conspiracy}

This class contains the subgraphs of statuses that spread conspiracy theories other than the ones related 


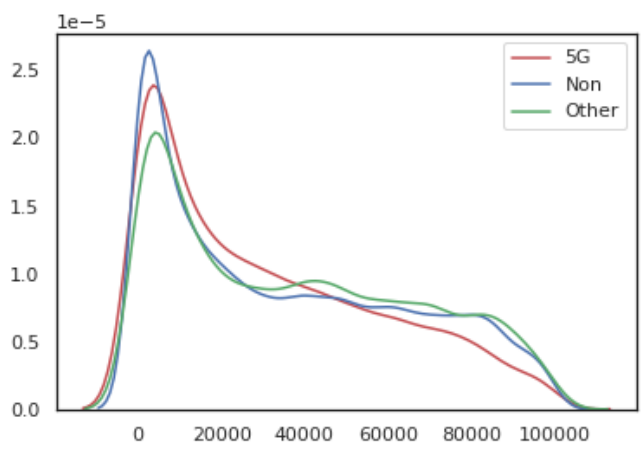

(a) Time difference in seconds

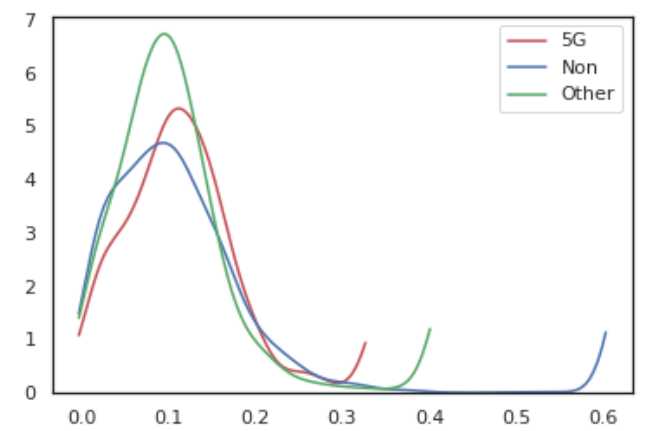

(c) Global CC

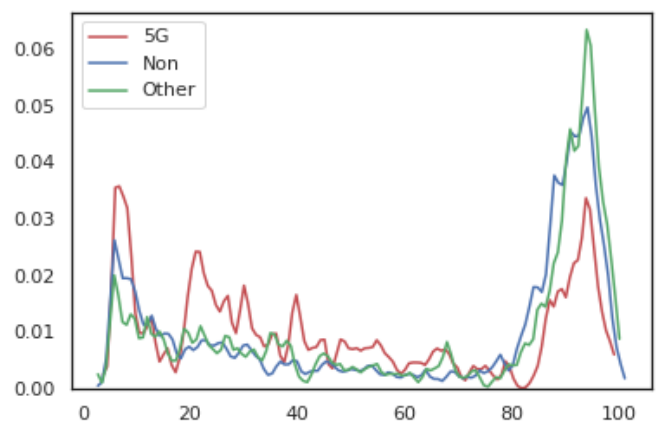

(e) Number of Nodes

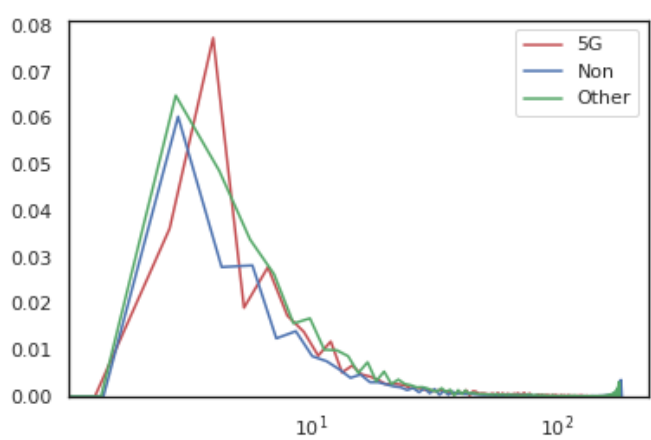

(b) Degree Distribution

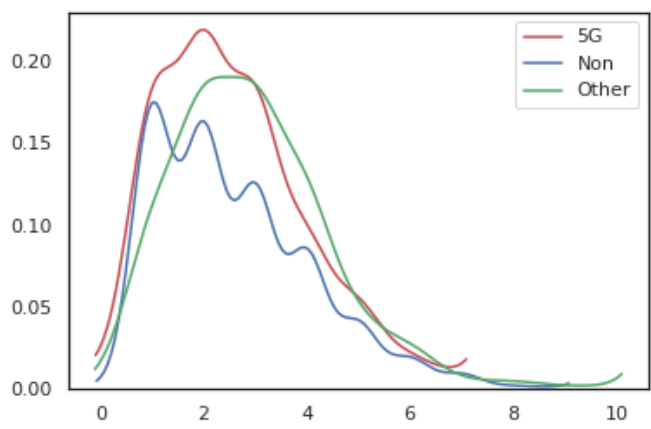

(d) Radius

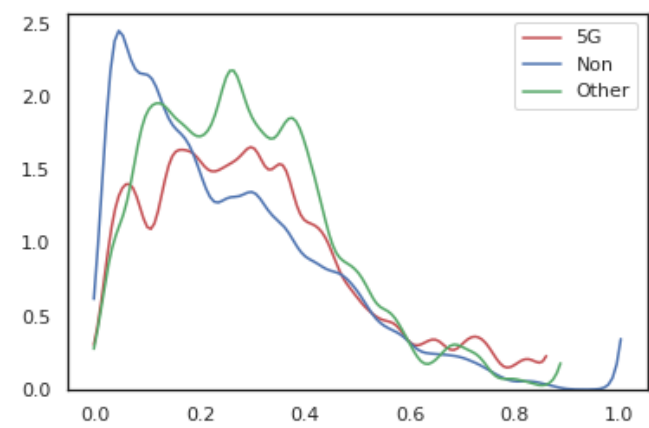

(f) Average Clustering Coefficient

Figure 2: Basic properties of the subgraphs by class. All key figures that can be seen here are normalized with the density fuction on the Y-axis. 
to $5 \mathrm{G}$. Such conspiracies include ideas about an intentional release of the virus, forced or harmful vaccinations, or the idea that the virus is a hoax. Because of the pre-selection explained in Section 5, all these conspiracies were tweets related to the COVID-19 pandemic. There are 596 subgraphs in this category.

\section{Non-Conspiracy}

This class contains the subgraphs of statuses not belonging to the previous two classes, including claims that $5 \mathrm{G}$ is harmful without linking it to COVID-19, as well as tweets claiming authorities are pushing for the installation of $5 \mathrm{G}$ the public is distracted by COVID19. Also, statuses pointing out the existence of conspiracy theories or mocking them fall into this class since they do not spread the conspiracy theories by inciting people to believe in them. The remaining 2490 subgraphs fall into this class, making it far larger than the other two.

Statuses that were not in English or could otherwise not be classified were discarded and replaced by new randomly selected statuses. For each valid status, we scraped a subgraph of the Twitter graph induced by the accounts that retweeted it, along with the account that tweeted the original status.

\section{INDUCED SUBGRAPHS}

We define the follower graph $G_{F}=(V, E)$ as the graph where $V$ represents the set of all Twitter users and $E$ the total of follower subscriptions. Let $T$ be the set of statuses. Each status $t_{i}$ has an author $a_{i} \in V$, a set of retweeters $R_{i} \subseteq V$, and a class label. The dataset contains the subgraphs of $G_{F}$ where each subgraph $H_{i}=\left(V_{i}, E_{i}\right)$ belongs to a specific status $t_{i}$. Let $V_{i}=\left\{a_{i}\right\} \cup R_{i}$ and $H_{i}=G_{F}\left[V_{i}\right]$ i.e. each such graph is the subgraph of $G_{F}$ induced by the author and all retweeters. Note that while the Twitter follower network changes constantly, $G_{F}$ is a single, static graph. Thus, if there is an edge $\left(v_{k}, v_{l}\right)$ between two vertices in some subgraph $H_{i}$, the same edge exists in all subgraphs that contain both vertices. Figure 1 shows an example of a subgraph derived from a Tweet. For each element in each set of retweeters $R_{i}$, the dataset

also contains the retweet time as the difference in seconds from the posting of the original status $t_{i}$. Thus, the author vertex implicitly has a retweet time of 0 . Note that this time is a property of the tweet, not the account. A vertex $v \in V$ can be contained in subgraphs and have a different retweet time in each of them.

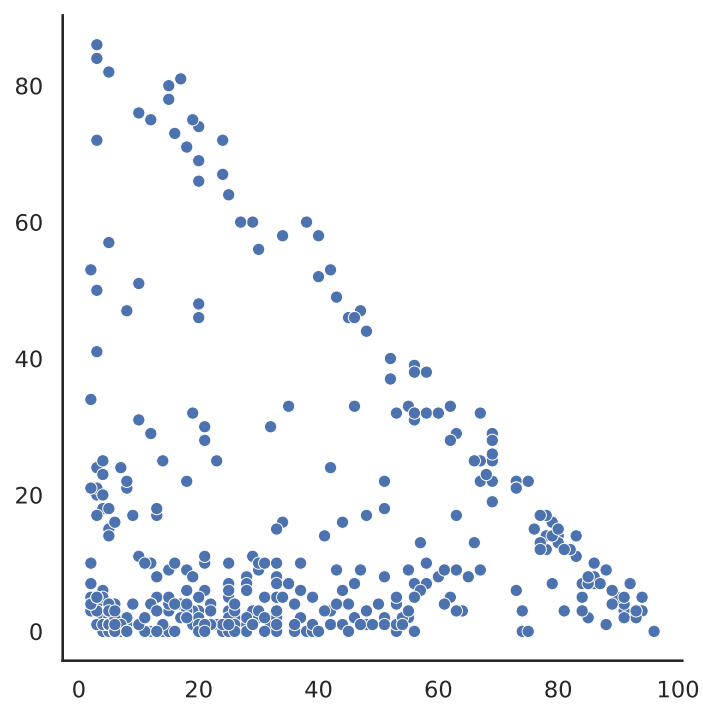

Figure 3: The size of the main component vs. the size of all other components for the 5G Conspiracy class.

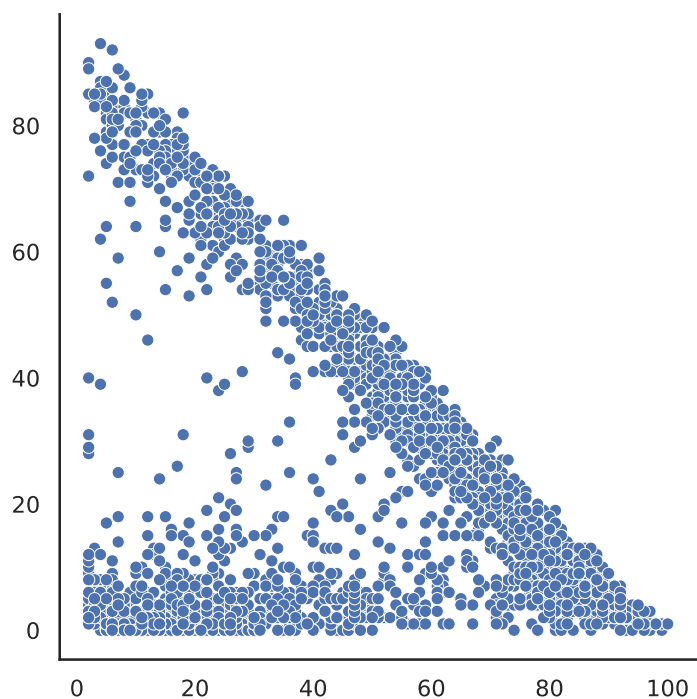

Figure 4: The size of the main component vs. the size of all other components for the Non-Conspiracy class.

Figure 2 shows the basic properties of the subgraphs in all three classes. While the difference is relatively small in most cases, we observe that graphs not associated with a conspiracy theory tend to be larger, have lower average degrees, higher clustering coefficient, and information spreads more slowly among them. All these measurements indicate that conspiracy theories are more likely to be shared among smaller, more densely connected groups of people, which, to a certain degree, is in line with the idea of echo chambers [Flaxman et al., 2016]. 


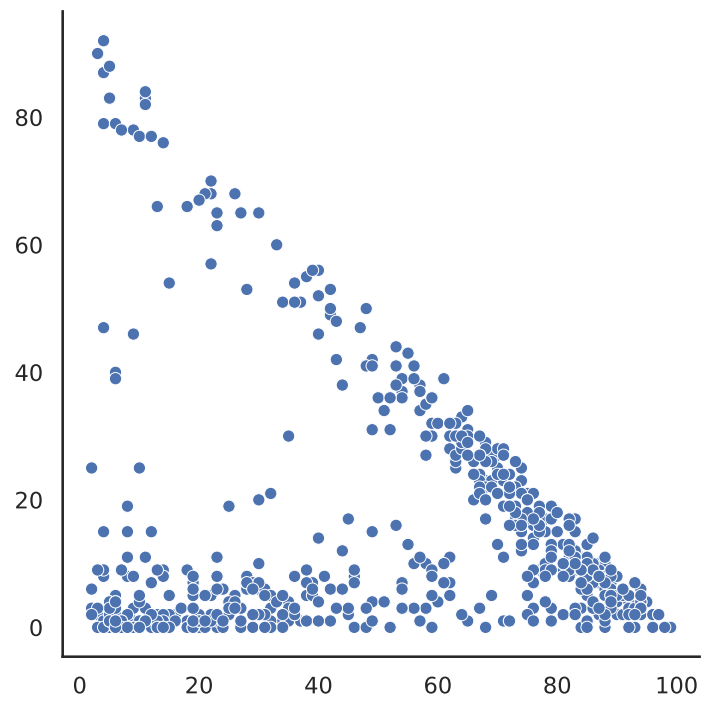

Figure 5: The size of the main component vs. the size of all other components for the Other Conspiracy class.

To further investigate the connectivity between the groups of retweeters, we also plot the size of the largest connected component in a subgraph against the number of nodes not in the largest component for each of the three classes. Results are shown in Figures 3, 4, and 5. In each plot, the X-axis represents the number of nodes in the main component and the Y-axis the number of other nodes in the subgraph. Since the subgraphs are limited to 100 nodes, the number are not percentages. Between the figures, we observe that the Non-Conspiracy class in Figure 4 contains a larger fraction of points on the diagonal, i.e. subgraphs with a relatively smaller main component. This further supports the observation that conspiracy tweets are more likely to spread in highly connected groups than other tweets. This is consistent with Figure 2c, which shows that the global clustering coefficient is lower in the same category.

\section{Limitations}

An important limitation is that the Retweet endpoint provided by the Twitter API, and along with it the size of the graphs, is limited to one hundred retweets. Thus, we have an accumulation of graphs having close to one hundred vertices, as shown in Figure 2e. Another aspect to consider is that the graphs themselves are distribution graphs, i.e. subgraphs of the Twitter follower graph. Thus, they include all follower edges, which do not necessarily represent the pathways via which the information spread since this cannot be inferred from the information available through the Twitter API. However, the dataset con- tains the direction of the spread of information indirectly, via the retweeting times. This allows excluding edges whose endpoint has a lower retweet time than the starting vertex.

Moreover, it is essential to note that Twitter's follower relation and the associated display of content on a user's newsfeed is not the only way content is distributed. Links to statuses can be shared outside of Twitter, for example, in news portals or in private chats. In case the distribution takes place outside of Twitter, connected components that are separate from the original status or even isolated nodes are formed (see Figure 1). We decided to provide these isolated components since they likely contain information that contributes to the classification. For methods that assume connected graphs and thus only consider the main connected component that contains the original status, they can be removed easily.

\section{DATASET STRUCTURE}

The dataset will be published as soon as author anonymity is lifted, using the format described below. The graphs are located individually in folders that are numbered in ascending order. Each folder contains three files. The edges.txt file contains a directed edge list source-id $\rightarrow$ target-id, the file nodes.csv contains an assignment from the node id to its properties (see Table 2), and the plot.png file contains a plot of the corresponding subgraph.

Table 2: The list of properties assigned to each sub graph node.

\begin{tabular}{|l|l|}
\hline id & $\begin{array}{l}\text { An anonymized id which remains the } \\
\text { same for all graphs in the datasets of } \\
\text { all categories. }\end{array}$ \\
\hline time & $\begin{array}{l}\text { The time difference in seconds from } \\
\text { each tweet to the original status. The } \\
\text { original status always has a difference } \\
\text { of 0 seconds to itself. }\end{array}$ \\
\hline friends & $\begin{array}{l}\text { The next greater power of two of the } \\
\text { follower count from the user profile } \\
\text { of the respective user. }\end{array}$ \\
\hline followers & $\begin{array}{l}\text { The next greater power of two of the } \\
\text { friend count from the user profile of } \\
\text { the respective user. }\end{array}$ \\
\hline
\end{tabular}

The number of nodes in the nodes.csv file does not necessarily match the number of nodes included in the edges.txt because nodes without any edges are not contained in the latter. The friends and followers counts are rounded to the next power of two to preserve anonymity. 


\section{BASELINE EXPERIMENTS}

The dataset aims to support the design of structurebased detection of misinformation, i.e., detection of misinformation tweets by analyzing the associated distribution graph. Naturally, this is a challenging task since there is no guarantee per se that the graphs associated with conspiracy theories differ from those that are not. Thus, in order to establish a baseline for the attainable accuracy, we present the results of three different classification approaches. The first two approaches, Naive Bayes and Random Forest, are used to learn classification based on manually extracted graph features. As a third approach, we use a graph neural network (GNN) to test whether the structure of the distribution graphs contains patterns aside from those implied by the explicit features that allow classification. We have performed the classification in the following variations to show how $5 \mathrm{G}$-specific misinformation relates to misinformation in general. In the first variant, we combined the graphs from the $5 G$ Conspiracy class with the graphs from the Other Conspiracy class to form the new class which we call General Conspiracy. The second variant contains only the classes $5 G$ Conspiracy and Non-Conspiracy, while the third variant contains only the classes Other Conspiracy and Non-Conspiracy. In the case of the first two simple classifications, we have added another variant in which we try to distinguish all three classes.

In order to train the Naive Bayes and the Random Forest classifier, we have extracted the following features from the subgraphs:

1. Number of Nodes: The number of nodes per graph. We count all nodes, including the isolated ones (see Figure 1 blue, red, purple, green).

2. Number of Edges: The number of directed edges for each graph. Thus, if two accounts follow each other, we count this as two edges.

3. Radius: The node corresponding to the original status of the subgraph. The radius of a distribution graph is the longest shortest path from the origin node. Each edge has length one.

4. Average Clustering Coefficient: The average clustering coefficient is defined as $C=\frac{\sum_{i=1}^{n} C_{i}}{n}$ where $n$ is the number of vertices and $C_{i}$ the Clustering Coefficient corresponding to node $i$.

For all experiments, we perform tenfold cross validation. In Table 3 we show precision, recall, F1 score, and Matthew correlation coefficient in addition to the accuracy. Both classifiers show a relatively similar behavior. As expected, distinguishing $5 G$ Conspiracy and Non-Conspiracy is the easiest case, while the multiclass problem is the hardest.
Table 3: Results of the feature-based classifiers using different performance metrics. PREC $=$ Precision, SENS = Recall, F1 = F1 Score, MCC = Matthew correlation coefficient

\begin{tabular}{|c|c|c|c|c|}
\hline Class & PREC & SENS & F1 & MCC \\
\hline \multicolumn{5}{|c|}{ Random Forest } \\
\hline \multicolumn{5}{|c|}{ General-Non } \\
\hline \multicolumn{5}{|c|}{ Correctly Classified Instances $68.1558 \%$} \\
\hline general & 0.415 & 0.269 & 0.327 & 0.136 \\
\hline non & 0.742 & 0.847 & 0.791 & 0.136 \\
\hline avg. & 0.649 & 0.682 & 0.658 & 0.136 \\
\hline \multicolumn{5}{|c|}{$5 G-$ Non } \\
\hline \multicolumn{5}{|c|}{ Correctly Classified Instances $83.4599 \%$} \\
\hline $5 G$ & 0.291 & 0.126 & 0.176 & 0.110 \\
\hline non & 0.870 & 0.950 & 0.908 & 0.110 \\
\hline avg. & 0.788 & 0.835 & 0.805 & 0.110 \\
\hline \multicolumn{5}{|c|}{ Other Non } \\
\hline \multicolumn{5}{|c|}{ Correctly Classified Instances $78.5159 \%$} \\
\hline other & 0.352 & 0.134 & 0.194 & 0.114 \\
\hline non & 0.820 & 0.941 & 0.876 & 0.114 \\
\hline avg. & 0.729 & 0.785 & 0.744 & 0.114 \\
\hline \multicolumn{5}{|c|}{ Multiclass } \\
\hline \multicolumn{5}{|c|}{ Correctly Classified Instances $66.8671 \%$} \\
\hline other & 0.240 & 0.114 & 0.155 & 0.055 \\
\hline non & 0.734 & 0.893 & 0.805 & 0.117 \\
\hline $5 \mathrm{G}$ & 0.246 & 0.108 & 0.150 & 0.094 \\
\hline avg. & 0.593 & 0.669 & 0.618 & 0.103 \\
\hline \multicolumn{5}{|c|}{ Naive Bayes } \\
\hline \multicolumn{5}{|c|}{ General - Non } \\
\hline \multicolumn{5}{|c|}{ Correctly Classified Instances $69.2153 \%$} \\
\hline general & 0.341 & 0.078 & 0.127 & 0.031 \\
\hline non & 0.717 & 0.939 & 0.813 & 0.031 \\
\hline avg. & 0.609 & 0.692 & 0.616 & 0.031 \\
\hline \multicolumn{5}{|c|}{$5 G-N o n$} \\
\hline \multicolumn{5}{|c|}{ Correctly Classified Instances $84.5304 \%$} \\
\hline $5 \mathrm{G}$ & 0.256 & 0.054 & 0.089 & 0.058 \\
\hline non & 0.863 & 0.974 & 0.915 & 0.058 \\
\hline avg. & 0.778 & 0.845 & 0.800 & 0.058 \\
\hline \multicolumn{5}{|c|}{ Other Non } \\
\hline \multicolumn{5}{|c|}{ Correctly Classified Instances $78.8723 \%$} \\
\hline other & 0.245 & 0.045 & 0.076 & 0.025 \\
\hline non & 0.809 & 0.967 & 0.881 & 0.025 \\
\hline avg. & 0.700 & 0.789 & 0.725 & 0.025 \\
\hline \multicolumn{5}{|c|}{ Multiclass } \\
\hline \multicolumn{5}{|c|}{ Correctly Classified Instances $69.0722 \%$} \\
\hline other & 0.200 & 0.034 & 0.057 & 0.013 \\
\hline non & 0.715 & 0.955 & 0.818 & 0.020 \\
\hline $5 \mathrm{G}$ & 0.221 & 0.037 & 0.063 & 0.046 \\
\hline avg. & 0.570 & 0.691 & 0.600 & 0.022 \\
\hline
\end{tabular}

We also perform a short test whether basic graph neural networks (GNNs) can provide even better ac- 
curacy. As there are numerous types of GNNs a full investigation would be outside the scope of this paper. We performed a comparison of a subset of the available state-of-the-art GNNs based on the paper by Errica et al. [Errica et al., 2020]. Among the tested alternatives, the Graph Isomorphism Network (GIN) [Xu et al., 2018] performed best.

GINs are relatively simple, but they are proven to be one of the most expressive architectures among the GNNs. Since we are only performing graph classification, we use the pooling variant of GIN to get a graph representation of the computed node embeddings. We perform a short hyperparameter optimization for the main task, which is separating the $5 \mathrm{G}$ from the non-conspiracy class. The results are listed in Table 4 .

\begin{tabular}{|l|l|l|l|}
\hline Batch Size & Units & Layers & Accuracy \\
\hline 32 & 32 & 3 & $64.2 \% \pm 8.4 \%$ \\
\hline 32 & 32 & 5 & $62.3 \% \pm 8.0 \%$ \\
\hline 32 & 64 & 2 & $64.8 \% \pm 7.2 \%$ \\
\hline 32 & 64 & 5 & $64.8 \% \pm 8.9 \%$ \\
\hline 128 & 32 & 3 & $\mathbf{6 7 . 4 \%} \pm \mathbf{9 . 0 \%}$ \\
\hline 128 & 32 & 5 & $62.7 \% \pm 7.4 \%$ \\
\hline 128 & 64 & 2 & $64.4 \% \pm 6.7 \%$ \\
\hline 128 & 64 & 5 & $66.7 \% \pm 7.9 \%$ \\
\hline
\end{tabular}

Table 4: Hyperparameter optimization for the GNN experiment on the $5 \mathrm{G}$ vs Non-Conspiracy classification problem.

Based on the accuracy, a network with 32 units, three layers, and a batch size of 128 emerged as the best alternative. This is understandable given the low average diameter of the graphs, as shown in Figure $2 d$. We also trained and evaluated the same network configuration for the other two classification task. The accuracy for separating Non-Conspiracy from the rest and for separating the Other Conspiracies class alone from Non-Conspiracy was $62.4 \%$ and $62.2 \%$ respectively. These results show that the simple GIN approach performs significantly worse than the other classifiers. Using Random Forest or Naive Bayes, structure-based classification of misinformation is possible. It remains to be seen whether the accuracy of the GNN based approach can be improved. This dataset aims to provide a basis for the development of more accurate classifiers.

\section{CONCLUSION}

We have presented a new dataset of Twitter graphs associated with the spread of misinformation related to COVID-19, particularly conspiracy theories connected to $5 \mathrm{G}$. This dataset can be used to train graph- based misinformation detection methods. The dataset is comparatively small, which is due to the effort required for manual labeling, and because very few such misinformation tweets have a substantial number of retweets. We have used basic classifiers to verify that ML-based classification is possible and to establish a baseline accuracy that more sophisticated systems can compare against. However, since the ultimate goal for such systems will be to moderate or at least flag content in social media, we believe that explainability will be a quality in addition to high accuracy, a consideration that was also pointed out in previous work [Reis et al., 2019].

In the future, we will pursue the approach of finding candidate misinformation tweets among the statuses that have sizeable subgraphs associated with them using natural language processing. The resulting candidate statuses can then be labeled manually. The labeling showed that the differences between misinformation spreading and other Tweets can be very subtle. Thus we believe that manual labeling remains necessary as current NLP methods are not capable of reliably identifying misinformation. However, such methods can be developed with the help of the labeled datasets. We assume that hybrid methods that combine graph and NLP based approaches will be the key to obtain reliable misinformation detection systems.

\section{REFERENCES}

Ahmed, S., Hinkelmann, K., and Corradini, F. (2019). Combining machine learning with knowledge engineering to detect fake news in social networks-a survey. In Proceedings of the AAAI 2019 Spring Symposium, volume 12 .

Ali, H. S. and Kurasawa, F. (2020). \#covid19: Social media both a blessing and a curse during coronavirus pandemic. https://bit.ly/3bjVQge.

Breiman, L. (2001). Random forests. Machine learning, 45(1):5-32.

Burkhardt, J. M. (2017). History of fake news. Library Technology Reports, 53(8):5-9.

Castelo, S., Almeida, T., Elghafari, A., Santos, A., Pham, K., Nakamura, E., and Freire, J. (2019). A topicagnostic approach for identifying fake news pages. In Companion Proceedings of The 2019 World Wide Web Conference, WWW '19, page 975-980, New York, NY, USA. Association for Computing Machinery.

Cui, L. and Lee, D. (2020). Coaid: Covid-19 healthcare misinformation dataset.

Cui, L., Seo, H., Tabar, M., Ma, F., Wang, S., and Lee, D. (2020). Deterrent: Knowledge guided graph attention network for detecting healthcare misinformation. In Proceedings of the 26th ACM SIGKDD International 
Conference on Knowledge Discovery \& Data Mining, KDD '20, page 492-502, New York, NY, USA. Association for Computing Machinery.

Dai, E., Sun, Y., and Wang, S. (2020). Ginger cannot cure cancer: Battling fake health news with a comprehensive data repository. In Proceedings of the International AAAI Conference on Web and Social Media, volume 14, pages $853-862$.

de Beer, D. and Matthee, M. (2020). Approaches to identify fake news: A systematic literature review. In Antipova, T., editor, Integrated Science in Digital Age 2020, pages 13-22, Cham. Springer International Publishing.

Dhoju, S., Main Uddin Rony, M., Ashad Kabir, M., and Hassan, N. (2019). Differences in health news from reliable and unreliable media. In Companion Proceedings of The 2019 World Wide Web Conference, pages 981-987.

Errica, F., Podda, M., Bacciu, D., and Micheli, A. (2020). A fair comparison of graph neural networks for graph classification. In International Conference on Learning Representations.

European External Action Service (EEAS) (2020). Disinformation can kill. https://bit. ly/32FKlwb.

Flaxman, S., Goel, S., and Rao, J. M. (2016). Filter Bubbles, Echo Chambers, and Online News Consumption. Public Opinion Quarterly, 80(S1):298-320.

Ghebreyesus, T. A. and Ng, N. (2020). How the who is leading the fight against coronavirus misinformation.

Ghenai, A. and Mejova, Y. (2018). Fake cures: user-centric modeling of health misinformation in social media. Proceedings of the ACM on human-computer interaction, 2(CSCW):1-20.

Hassan, N., Arslan, F., Li, C., and Tremayne, M. (2017). Toward automated fact-checking: Detecting checkworthy factual claims by claimbuster. In Proceedings of the 23rd ACM SIGKDD International Conference on Knowledge Discovery and Data Mining, KDD '17, page 1803-1812, New York, NY, USA. Association for Computing Machinery.

Horne, B. D. and Adali, S. (2017). This just in: Fake news packs a lot in title, uses simpler, repetitive content in text body, more similar to satire than real news. arXiv preprint arXiv: 1703.09398.

Hosseinimotlagh, S. and Papalexakis, E. E. (2018). Unsupervised content-based identification of fake news articles with tensor decomposition ensembles. In Proceedings of the Workshop on Misinformation and Misbehavior Mining on the Web (MIS2).

Howell, L. (2013). Digital wildfires in a hyperconnected world. https://bit.ly/2GiEF4f.

Huang, Q., Zhou, C., Wu, J., Wang, M., and Wang, B. (2019). Deep structure learning for rumor detection on twitter. In 2019 International Joint Conference on Neural Networks (IJCNN), pages 1-8. IEEE.

Kersting, K., Kriege, N. M., Morris, C., Mutzel, P., and Neumann, M. (2016). Benchmark data sets for graph kernels. http://graphkernels.cs.tu-dortmund. de.
Le, T., Wang, S., and Lee, D. (2020). Malcom: Generating malicious comments to attack neural fake news detection models. arXiv preprint arXiv:2009.01048.

Ma, J., Gao, W., and Wong, K.-F. (2019). Detect rumors on twitter by promoting information campaigns with generative adversarial learning. In The World Wide Web Conference, pages 3049-3055.

Pérez-Rosas, V., Kleinberg, B., Lefevre, A., and Mihalcea, R. (2018). Automatic detection of fake news. In Proceedings of the 27th International Conference on Computational Linguistics, pages 3391-3401, Santa Fe, New Mexico, USA. Association for Computational Linguistics.

Reis, J. C., Correia, A., Murai, F., Veloso, A., and Benevenuto, F. (2019). Explainable machine learning for fake news detection. In Proceedings of the 10th ACM Conference on Web Science, pages 17-26.

Salem, F. K. A., Al Feel, R., Elbassuoni, S., Jaber, M., and Farah, M. (2019). Fa-kes: A fake news dataset around the syrian war. In Proceedings of the International AAAI Conference on Web and Social Media, volume 13, pages 573-582.

Schroeder, D. T., Pogorelov, K., and Langguth, J. (2019). Fact: a framework for analysis and capture of twitter graphs. In 2019 Sixth International Conference on Social Networks Analysis, Management and Security (SNAMS), pages 134-141. IEEE.

Shu, K., Mahudeswaran, D., Wang, S., Lee, D., and Liu, H. (2018). Fakenewsnet: A data repository with news content, social context and dynamic information for studying fake news on social media. arXiv preprint arXiv:1809.01286, 8.

Sivasangari, V., Anand, P., and Santhya, R. (2018). A modern approach to identify the fake news using machine learning. Int. J. Pure Appl. Math, 118(20):10.

Wang, W. Y. (2017). " liar, liar pants on fire": A new benchmark dataset for fake news detection. arXiv preprint arXiv:1705.00648.

Xu, K., Hu, W., Leskovec, J., and Jegelka, S. (2018). How powerful are graph neural networks? CoRR, abs/1810.00826.

Yang, Y., Zheng, L., Zhang, J., Cui, Q., Li, Z., and Yu, P. S. (2018). Ti-cnn: Convolutional neural networks for fake news detection.

Zhang, C., Gupta, A., Kauten, C., Deokar, A., and Qin, X. (2019). Detecting fake news for reducing misinformation risks using analytics approaches. European Journal of Operational Research, 279. 